\title{
Spectral Determination of Nonlinear System Parameters
}

\author{
Adil Brouri ${ }^{1, *}$, Mohamed Benyassi ${ }^{2}$ \\ ${ }^{1}$ ENSAM, IMSM, L2MC, AEEE Department, Moulay Ismail University, Meknès, Morocco \\ ${ }^{2}$ ESTM, LEM2A, Electrical Engineering Department, Moulay Ismail University, Meknès, Morocco
}

\begin{abstract}
In this paper we propose an identification method of nonlinear system. This later can be structured by Wiener models. The determination of nonlinear system parameters can be done using spectral analysis. The system nonlinearity is allowed to be noninvertible general shape nonlinearity but it must be approximated by a polynomial function. The polynomial degree $\mathrm{n}$ can vary from one interval to another. The linear dynamic element is not-necessarily parametric but BIBO stable. In this work, a spectral method is developed allowing the estimates of the complex frequency gain as well as the estimates of nonlinear block parameters the identification method is built using one stage.
\end{abstract}

\section{Introduction}

This nonlinear system identification has been an active research area, especially over the last two decade [1]-[3]. A Wiener model is one of most popular models. It consists of linear element followed by nonlinearity block (Fig. 1). This model is more difficult than Hammerstein model (nonlinearity element followed by linear dynamic element). The usefulness of this nonlinear model has practically been confirmed in various domains fields [4] - [6].

In the last decades, several studies on identification problem have been made [7]-[10]. Roughly, these solutions can be classified into four categories. The recursive method [11], blind-identification methods [12], the stochastic methods [13]-[14], and the frequency solution [15]-[18].

In the present work, Wiener system identification is addressed in the case where the linear dynamic block is nonparametric and of unknown structure. This later is assumed to be BIBO stable. This assumption is commonly used in open loop identification case. On the other hand, it is assumed that the system nonlinearity $f($. can be approximated by a polynomial function. The polynomial degree $n$ can vary from one interval to the author's knowledge; the identification approach is performed using only one stage unlike most of other methods [19].

The suggested identification approach involves periodic input signals. Then, in the steady state, the undisturbed system output $w(t)$ is also periodic signal of the same period of $u(t)$ (Fig. 1). Accordingly, the internal signal $w(t)$ is decomposable of Fourier series. The nonlinear system parameters (i.e. the nonlinearity block parameters as well as the linear subsystem parameters) can be determined using $u(t)$ and the system output.

The remaining parts of paper are organized as follows: the identification problem is formulated in
Section 2; the identification method of linear and nonlinear elements is presented in Section 3; the identification method performances are illustrated by simulation example presented in Section 4. Except of this assumption, the nonlinear element can be of general shape and noninvertible.

Presently, a spectral analysis method is developed allowing an accurate estimates of complex frequency gain $G(j \omega)$ (i.e. the gain modulus $|G(j \omega)|$ and the phase $\angle G(j \omega)$ ) for any frequency $\omega$ as well as the estimates of system nonlinearity parameters.

To the author's knowledge, the identification approach is performed using only one stage unlike most of other methods [19].

The suggested identification approach involves periodic input signals. Then, in the steady state, the undisturbed system output $w(t)$ is also periodic signal of the same period of $\mathrm{u}(\mathrm{t})$ (Fig. 1). Accordingly, the internal signal $w(t)$ is decomposable of Fourier series. The nonlinear system parameters (i.e. the nonlinearity block parameters as well as the linear subsystem parameters) can be determined using $u(t)$ and the system output.

The remaining parts of paper are organized as follows: the identification problem is formulated in Section 2; the identification method of linear and nonlinear elements is presented in Section 3; the identification method performances are illustrated by simulation example presented in Section 4.

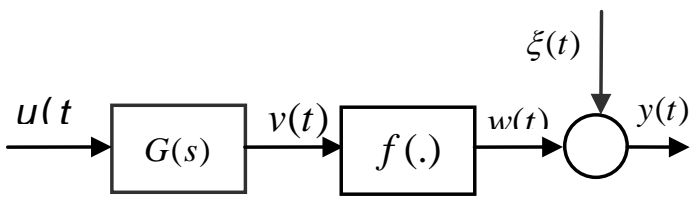

Fig. 1. Nonlinear system structured by Wiener model

\footnotetext{
* Corresponding author:a.brouri@ensam-umi.ac.ma
} 


\section{Identification Problem Statement}

Use Wiener models consist of series connection of linear block $G(s)$ and nonlinear static element $f($.). Presently, the linear dynamic block can be nonparametric and of unknown structure. Furthermore, the identification schema is built up in open loop. Then, the linear element is only supposed to be BIBO stable. The system nonlinearity is allowed to be noninvertible but can be approximated by polynomial function. The polynomial degree $n$ can varies from one interval to another.

One other hand, the nonlinear Wiener system (Fig. 1) under study can be analytically described by the following equations:

$$
v(t)=g(t) * u(t)
$$

where * refers to the convolution operator and:

$$
g(t)=\mathrm{L}^{-1}(G(s))
$$

The notation $\mathrm{L}^{-1}$ denotes Laplace transform-inverse. The internal signals $v(t)$ and $w(t)$ are related by the following equation:

$$
w(t)=f(v(t))
$$

Finally, the system output $y(t)$ can be expressed as follows:

$$
y(t)=w(t)+\xi(t)
$$

Using the fact that the system nonlinearity $f($.)can be modelled within any interval by polynomial function of degree $\mathrm{n}$. Then, one immediately has:

$$
f(x)=a_{0}+a_{1} x+\ldots+a_{n} x^{n}=\left[\begin{array}{lll}
a_{0} & \cdots & a_{n}
\end{array}\right]\left[\begin{array}{c}
1 \\
x \\
\vdots \\
x^{n}
\end{array}\right]=\theta^{T} X
$$

where $\theta=\left[\begin{array}{lll}a_{0} & \cdots & a_{n}\end{array}\right]^{T}$ is parameters vector of the nonlinearity $f($.$) .$

On the other hand, it is important to emphasize that, this problem identification does not have a unique solution (solution plurality). Indeed, if the couple $(G(s), f(v))$ is solution of this problem, then any couple of the form $(G(s) / k, f(k v))$ is also a model, for any $k \neq 0$. In this respect, the question that arises is how to choose the factor $k$ ?

This question will be dealt in the next section.

The aim of this study is to determine an accurate estimate of the parameters vector $\theta$ of the nonlinearity $f($.$) as well as the complex frequency gain G(j \omega)$ for any frequency $\omega$ (i.e. the phase $\varphi(\omega)=\arg (G(j \omega))=\angle G(j \omega)$ and the modulus gain $|G(j \omega)|)$. Roughly, two statements can arise. Firstly, if the linear element is parametric, then this latter has a limited number $m$ of unknown parameters. The identification of this latter consists thus to estimate the complex frequency gain $G(j \omega)$ for $m$ frequencies $\omega \in\left\{\omega_{1}, \ldots, \omega_{m}\right\}$, arbitrarily chosen by the user. The objective in the second case (i.e. the linear block is nonparametric) is to determine the complex gain $G(j \omega)$ for any frequency $\omega$.

Presently, the linear subsystem is nonparametric and can be of unknown structure. Finally, note that the extrainput $\xi(t)$ is counted for external noise. It is supposed to be a random ergodic sequence of zero-mean and satisfying the stationarity property. Then, all the internalsignals $v(t)$ and $w(t)$ are not accessible to measurement; the input and output of the system $(u(t)$ and $y(t))$ are the only measurable signals.

\section{Frequencies - Domain Identification}

The frequency identification of the this nonlinear system (Wiener model), characterized by (1)-(4), amounts to accurately estimating the nonlinear block parameters as well as the frequency complex gain $G(j \omega)$ for a set of frequencies $\left\{\omega_{1}, \ldots, \omega_{m}\right\}$.

The proposed frequency solution, allowing to estimate the nonlinear system parameters, is based on input sine signals:

$$
u(t)=U \sin (\omega t)
$$

where $\omega \in\left\{\omega_{1}, \ldots, \omega_{m}\right\}$. It readily follows from (1a-b)-

(5) that, the inner signal $v(t)$ can be expressed as (in steady state):

$$
v(t)=U|G(j \omega)| \sin (\omega t+\varphi(\omega))
$$

Accordingly, one immediately gets using (3) and (6):

$$
w(t)=\theta^{T}\left[\begin{array}{c}
1 \\
v(t) \\
\vdots \\
v(t)^{n}
\end{array}\right]=\sum_{k=0}^{n} a_{k}(U|G(j \omega)|)^{k} \sin ^{k}(\omega t+\varphi(\omega))
$$

The unknown parameters in (7) are the vector $\theta=\left[\begin{array}{lll}a_{0} & \cdots & a_{n}\end{array}\right]^{T}$ and the linear block parameters $(|G(j \omega)|, \varphi(\omega))$. On the other hand, one has using the power reduction formulas: 


$$
\begin{aligned}
& \sin ^{2 l}(\omega t+\varphi(\omega))=\frac{1}{2^{2 l}} C_{l}^{2 l}+ \\
& \frac{2}{2^{2 l}} \sum_{i=0}^{l-1}(-1)^{l-i} C_{i}^{2 l} \sin \left(2(l-i)(\omega(t)+\varphi(\omega))+\frac{\pi}{2}\right) \\
& \sin ^{2 l+1}(\omega t+\varphi(\omega))= \\
& \frac{1}{2^{2 l}} \sum_{i=0}^{l-1}(-1)^{l-i} C_{i}^{2 l+1} \sin ((2 l+1-2 i)(\omega(t)+\varphi(\omega)))
\end{aligned}
$$

Then, it is readily seen using (7) and (8a-b) that, the undisturbed output signal $w(t)$ can be rewritten as:

$$
w(t)=\sum_{k=0}^{n} S_{k}(\theta,|G(j \omega)|) \sin \left(k \omega t+\lambda_{k}(\varphi(\omega))\right)
$$

This mean that $w(t)$ is equivalent to sum of $\mathrm{n}$ sine signals, where the amplitude $S_{k}(k=1, \cdots, n)$ depends on the parameters $\left(a_{0}, \cdots, a_{n}\right)$ and the modulus gain $|G(j \omega)|$; as for the phase $\lambda_{k}(k=1, \cdots, n)$, it depends only on the linear element argument $\varphi(\omega)$. Accordingly, one immediately gets using (3) and (9):

$$
y(t)=\sum_{k=0}^{n} S_{k}(\theta,|G(j \omega)|) \sin \left(k \omega t+\lambda_{k}(\varphi(\omega))\right)+\xi(t)
$$

This results shows that, the undisturbed output signal $w(t)$ (not accessible to measurement) is periodic of the same period $2 \pi / \omega$ of input $u(t)$. Likewise, the spectrum of inner signal $w(t)$ is characterized by $n$ components of frequency $k \omega(k=1, \cdots, n)$ and a DC component (see (9)). It follows using the measurement of $w(t)$ that, an accurate estimate of the frequency component amplitudes $S_{k}(k=0, \cdots, n)$ as well as of the phases $\lambda_{k}($.$) . In this respect, it is interesting to emphasize$ that the undisturbed output $w(t)$ is not accessible to measurement and the output signal $y(t)$ (measurable) is $w(t)$ mixed up to noise $\xi(t)$.

Fortunately, such an accurate estimation can be available thanks to the ergodicity property of the noise signal $\xi(t)$ and the steady-state periodic nature of the inner signal $w(t)$. The ergodicity allows the substitution of arithmetic averages to probabilistic means, making simpler forthcoming developments. These remarks suggest that an accurate estimate $\hat{w}(t)$ of $w(t)$ can be obtained using the following periodical averaging:

$$
\begin{array}{cc}
\hat{w}(t)=\frac{1}{N} \sum_{k=1}^{N} y(t+k T) & \text { for } \quad t \in[0 T) \\
\hat{w}(t+k T)=\hat{w}(t) \quad \text { for } \quad k=1,2, \ldots
\end{array}
$$

where $N$ is any sufficiently large integer. It is readily shown that, the suggested estimator (11a-b) is consistent, i.e. the inner signal estimate $\hat{w}(t)$ converges with probability 1 to the true signal $w(t)$. Indeed, one immediately gets using (3) and (11a-b):

$$
\hat{w}(t)=\frac{1}{N} \sum_{k=1}^{N} w(t+k T)+\frac{1}{N} \sum_{k=1}^{N} \xi(t+k T) \forall t
$$

Then, this latter becomes using the periodic nature of $w(t)$ :

$$
\hat{w}(t)=w(t)+\frac{1}{N} \sum_{k=1}^{N} \xi(t+k T) \forall t
$$

Finally, the point is that the last term in (13) boils down to zero (w.p.1) using the ergodicity property of the noise $\xi(t)$. One gets thus from (13) the following convergence:

$$
\lim _{N \rightarrow \infty} \hat{w}(t)=w(t) \quad \text { whatever } t
$$

On the other hand, it is important to point that, the determination of spectrum of the signal $w(t)$ using (11ab) allows to estimate the DC component $S_{0}$, the frequency harmonic amplitudes $S_{k}(k=1, \cdots, n)$, and the component phases $\lambda_{k}(k=1, \cdots, n)$.

Therefore, it follows from the DC value $S_{0}$ and the harmonic amplitudes $S_{k}(k=1, \cdots, n)$ that, $(n+1)$ equations are generated that involve $(n+2)$ unknown parameters, i.e. the nonlinearity coefficients $\left(a_{0}, \cdots, a_{n}\right)$ and the modulus gain $\left|G\left(j \omega_{l}\right)\right|$ (where $\omega_{l} \in\left\{\omega_{1}, \ldots, \omega_{m}\right\}$ ). One has thus fewer equations than unknown parameters. At the same time using the component phases $\lambda_{k}$, only one unknown is involved, i.e. the linear element argument $\varphi\left(\omega_{l}\right)$ where $\omega_{l} \in\left\{\omega_{1}, \ldots, \omega_{m}\right\}$. Then, for any input signal (5) of frequency $\omega_{l} \in\left\{\omega_{1}, \ldots, \omega_{m}\right\}$, the phase of linear block $\varphi\left(\omega_{l}\right)$ can be easily determined using the phase estimate of any harmonic component $\lambda_{k}$. Accordingly, to resolve this estimation problem it is necessary to generate more equations involving the nonlinearity parameters $\left(a_{0}, \cdots, a_{n}\right)$ and the modulus gain of linear element. For convenience, the system is excited by the input sine signal (5) with another frequency $\omega_{p} \in\left\{\omega_{1}, \ldots, \omega_{m}\right\}$ and $\omega_{p} \neq \omega_{l}$.

Therefore, it follows using these two experiments that, $2(n+1)$ equations are generated that involve $(n+3)$ unknowns, i.e. the nonlinearity block parameters $\left(a_{0}, \cdots, a_{n}\right)$ and the modulus gains $\left(\left|G\left(j \omega_{l}\right)\right|,\left|G\left(j \omega_{p}\right)\right|\right)$. Finally, an accurate estimate of nonlinear system parameters can be determined 


\section{Conclusion}

Equations Presently, the problem of nonlinear system identification is dealt. The nonlinear system is described by Wiener model. This identification problem is coped in the frequency-domain using the identification solution described in Section 3. Then, the identification algorithm is built up using a simple sine signals. It is interesting to point out that, the parameters of linear and nonlinear elements are determined using only one stage.

\section{References}

1. A. Brouri, L. Kadi, S. Slassi, "Frequency identification of Hammerstein-Wiener systems with Backlash input nonlinearity", Int. J. of Control, Automation \& Systems, 15, No: 5, pp. 2222-2232, (2017).

2. A. Brouri, F. Giri, Y. Rochdi, F.Z. Chaoui, "Frequency identification of nonparametric Hammerstein systems with backlash nonlinearity", American Control Conference, San Francisco, CA, USA, June 29 - July 01,pp. 657-662, (2011).

3. M. Benyassi, A. Brouri, "Identification of Nonlinear Systems Having Nonlinearities at Input and Output", 2017 European Conference on Electrical Engineering and Computer Science (EECS), Bern, Switzerland, pp. 311-313, (2017).

4. A. Brouri, L. Kadi, S. Slassi, " Identification of Nonlinear Systems ", 2017 European Conference on Electrical Engineering and Computer Science (EECS), Bern, Switzerland, pp. 286-288, (2017).

5. L. Kadi, A. Brouri, "Numerical Modeling of a Nonlinear Four-phase Switched Reluctance Machine", IRSEC'17, IEEE, Tanger, Morocco, Dec 04-07, 2017.

6. A. Kalafatis, L. Wang, W.R. Cluett, "Identification of time-varying $\mathrm{pH}$ processes using sinusoidal signals," Automatica, 41, pp. 685-691, (2005).

7. A. Brouri, L. Kadi, "A Contribution on the Identification of Nonlinear Systems", 5th Inter. Conf. of Control, Decision \& Inf. Tech. (CoDIT'18), Thessaloniki, Greece, Apr 10-13, pp. 605-510, (2018).

8. A. Brouri, T. Rabyi, A. Ouannou, "Identification of Nonlinear Systems With Hard Nonlinearity", CoDIT'18, Thessaloniki, Greece, Apr 10-13, pp. 506-511, (2018).

9. A. Brouri, F.Z. Chaoui, O. Amdouri, F. Giri, "Frequency Identification of Hammerstein-Wiener Systems with Piecewise Affine Input Nonlinearity", 19th IFAC World Congress, Cape Town, South Africa, August 24-29, pp. 10030-10035, (2014).

10. A. Brouri, "Identification of Nonlinear Systems", AIP Conference Proceeding, 1836, 020031, (2017).

11. D. Westwick and M. Verhaegen, "Identifying MIMO Wiener systems using subspace model identification methods", Signal Processing, 52, pp. 235-258, 1996.

12. L. Vanbeylen, R. Pintelon, J. Schoukens, "Blind Maximum-likelihood Identification of Wiener systems", IEEE Trans. on Sig. Proc., 57, No:8, pp. 3017-3029, (2009).

13. W. Greblicki and M. Pawlak, "Nonparametric System Identification", Cambridge University Press, UK, (2008).

14. Z. Hasiewicz and G. Mzyk, "Hammerstein system identification by non-parametric instrumental Variables", International Journal of Control, vol. 82, No: 3, pp. 440-455, (2009).

15. A. Brouri, "Frequency identification of Hammerstein-Wiener systems with Backlash input nonlinearity", W. TRANSACT. on SYST. \& CONT., 12, pp. 82-94, (2017).

16. A. Brouri, "Wiener-Hammerstein Models Identification", Int. Journal of Math. Mod. \& Meth. in Applied Sc., 10, pp. 244-250, (2016).

17. A. Brouri, S. Slassi, "Identification of Nonlinear Systems Structured by Wiener-Hammerstein Model", International Journal of Electrical and Computer Engineering, 6, No:1, pp. 167-176, (2016).

18. A. Brouri, F. Giri, F. Ikhouane, F.Z. Chaoui, O. Amdouri, " Identification of Hammerstein-Wiener Systems with Backlask Input Nonlinearity Bordered By Straight Lines", 19th IFAC World Congress, Cape Town, South Africa, August 24-29, pp. 475480, (2014).

19. A. Brouri, Y. Rochdi, J.B. Gning, F. Giri, F.Z. Chaoui, "Frequency identification of Hammerstein systems with switch memory nonlinearities", in IFAC Proceedings, 18, pp. 13942-13947, (2011).

20. Luigi T. De Luca, Propulsion physics (EDP Sciences, Les Ulis, 2009)

21. F. De Lillo, F. Cecconi, G. Lacorata, A. Vulpiani, EPL, 84 (2008) 